\title{
Measurement of the CP structure of the Yukawa interaction in Higgs boson decays to $\tau$ leptons
}

\author{
Andrea Cardini ${ }^{a, *}$ on behalf of the CMS collaboration \\ ${ }^{a}$ Deutsches Elektronen-Synchrotron, \\ Notkestraße 85, Hamburg, Germany \\ E-mail: andrea.cardini@cern.ch
}

The discovery of the Higgs boson in 2012 by the CMS and ATLAS collaborations marked the start of the exploration of the Higgs sector of particle physics. The properties of the Higgs sector under CP symmetry have been investigated mostly in its couplings to gauge bosons. With the full Run 2 data-taking period it became possible to study the CP properties of the Yukawa coupling of the Higgs to fermions, and in particular to tau leptons. This was done by reconstructing the decay planes of the two tau leptons and measuring their angular correlation. The measured mixing angle between CP-even and CP-odd couplings is $(4 \pm 17)^{\circ}$ and is consistent with the Standard Model prediction of a pure $\mathrm{CP}$-even coupling and allows to constrain the allowed phase space for possible BSM scenarios. A pure CP-odd hypothesis is excluded at $99.7 \%$ confidence level.

The Ninth Annual Conference on Large Hadron Collider Physics - LHCP2021

7-12 June 2021

Online

${ }^{*}$ Speaker 


\section{Introduction}

The standard model of particle physics predicts the existence of one Higgs boson with spinparity $J^{P}=0^{+}[1,2]$. Any deviation from this prediction would lead to an additional source of $\mathrm{CP}$ violation. Investigating the $\mathrm{CP}$ properties of the Higgs boson is done by investigating how it behaves under parity symmetry and charge conjugation. In other words we want to observe the Higgs boson through a "CP mirror". CP violation in the Higgs sector can be investigated in:

- coupling to vector bosons (direct coupling to $\mathrm{Z}$ and $\mathrm{W}$ bosons in particular),

- Yukawa couplings:

- gluon gluon fusion via top quark loop and top-associated production,

- Higgs decays to $\tau$ leptons.

The poster associated to these proceedings aims at discussing the search for CP violation in the $H \rightarrow \tau \tau$ process.

\section{CP mixing in Yukawa coupling}

The Yukawa Lagrangian for the Higgs coupling to $\tau$ leptons can be written to account for an admixture of CP-even and CP-odd couplings at leading order:

$$
\mathcal{L}_{Y, \tau}=-\frac{m_{\tau}}{v} \bar{\tau}\left(\kappa_{\tau}+i \gamma^{5} \tilde{\kappa}_{\tau}\right) H \tau
$$

where $\kappa_{\tau}\left(\tilde{\kappa}_{\tau}\right)$ represents the CP-even(-odd) coupling, $\tau(\tilde{\tau})$ the spinor of the tau particle (antiparticle), $m_{\tau}$ the tau lepton mass and $H$ and $v$ the excitation of the Higgs field and its vacuum expectation value. The $\mathrm{CP}$-mixing can be encoded in the two couplings via the CP-mixing angle $\varphi_{\tau \tau}$ :

$$
\begin{aligned}
& \kappa=\sqrt{\mu^{\tau \tau}} \cos \left(\varphi_{\tau \tau}\right), \\
& \tilde{\kappa}=\sqrt{\mu^{\tau \tau}} \sin \left(\varphi_{\tau \tau}\right),
\end{aligned}
$$

with $\mu^{\tau \tau}$ representing the overall coupling modifier for the $H \rightarrow \tau \tau$ branching fraction. Due to the correlation between the $\tau$ spin and the momenta of its decay products, the cross section $\sigma$ for the $H \rightarrow \tau \tau$ process assumes a sinusoidal dependence with respect to the angle between the decay plains of the two $\tau$ leptons, i.e. the acoplanarity angle $\left(\varphi_{C P}\right)$ :

$$
\frac{\mathrm{d} \sigma}{\mathrm{d} \varphi_{C P}}=\text { const }-\cos \left(\varphi_{C P}-2 \varphi_{\tau \tau}\right)
$$

As shown in Fig. 1 the distribution is affected by a phase-shift in the presence of CP-mixing.

\section{The acoplanarity angle}

The acoplanarity angle is defined as the angle spanning between the decay planes of the two $\tau$ leptons and is depicted in the left part of Fig. 1. As the correlation between the $\tau$ lepton spin and the momenta of its decay products can vary depending on the decay channel [3], it is defined using different vectors in each decay mode. 

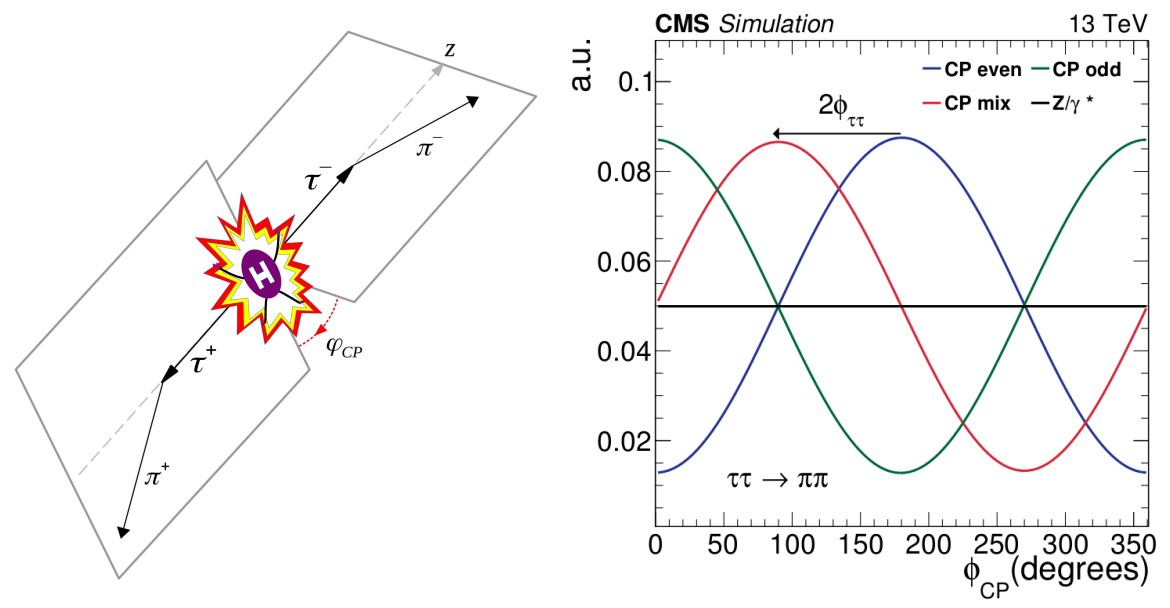

Figure 1: Left: Schematic depiction of the acoplanarity angle definition for a Higgs boson (depicted in cartoon form) decaying into two $\tau$ leptons, further decaying into one charged pion each. Right: Acoplanarity angle distribution in the same final state [4]. The distributions are shown for simulated $H \rightarrow \tau \tau$ processes, according to three different CP hypotheses. The acoplanarity distribution is shown for the DY process in gray and appears as a flat line.

In the analysis described in [4] the following $\tau$ decays have been considered ${ }^{1}: \tau^{ \pm} \rightarrow \mu^{ \pm}$, $\tau \pm \rightarrow \pi^{ \pm}, \tau^{ \pm} \rightarrow \rho \rightarrow \pi^{ \pm} \pi^{0}, \tau^{ \pm} \rightarrow a_{1}^{ \pm} \rightarrow \pi^{ \pm} \pi^{0} \pi^{0}, \tau^{ \pm} \rightarrow a_{1}^{ \pm} \rightarrow \pi^{ \pm} \pi^{ \pm} \pi^{\mp}$. Hadronic $\tau$ decays have been identified using the DeEPTAU algorithm [5], while the identification of the decay channel was performed with the MVA-DM algorithm [6]. When mesonic resonances are present in the decay chain, the acoplanarity angle is defined using the momenta of the charged and neutral pions in the decay. The impact parameter of charged particles is instead used for $\tau$ decays to a muon or a charged pion.

\section{Identification of Higgs decays}

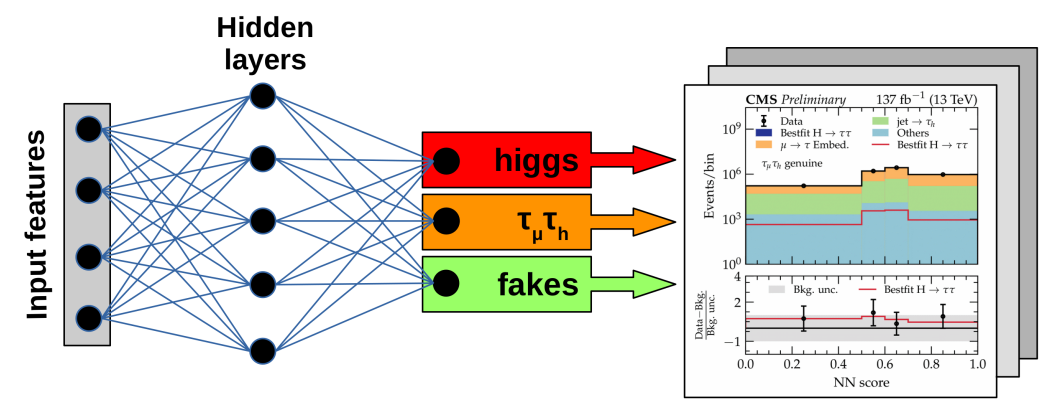

Figure 2: Schematic depiction of the machine learning algorithm used to identify Higgs decays [3].

The identification of Higgs boson decays to $\tau$ leptons was performed using multivariate analysis (MVA) techniques: a boosted decision tree (BDT) for the fully hadronic final state, and a neural

\footnotetext{
${ }^{1}$ The neutrinos have been removed from the decay chain to simplify the notation.
} 
network (NN) for the semileptonic one. As shown in Fig. 2 the MVA algorithms are defined with three output classes: one for the Higgs boson (higgs), one for processes involving final states with two genuine $\tau$ leptons $\left(\tau_{\mu} \tau_{\mathbf{h}}\right)$ and one for jets or leptons misidentified as hadronically decaying $\tau$ leptons (fakes).

\section{Extraction of the $\mathrm{CP}$ mixing angle and results}

The CP mixing angle $\varphi_{\tau \tau}$ is extracted from a parametric fit of a $H \rightarrow \tau \tau$ template, which considers three distinct production mechanisms for the Higgs boson: the gluon-gluon fusion, the vector boson fusion and the Higgs-strahlung. The fit is performed simultaneously in the signal category, split by $\tau$ decay channels, and the two background categories using the full statistics collected during the Run 2 data-taking period.

\section{Results}

The left part of Fig. 3 shows the weighted distribution of the acoplanarity angle. The recorded data is consistent with the SM expectation of a pure CP-even Yukawa coupling. The measured CP mixing angle is

$$
\left.\varphi_{\tau \tau}=(4 \pm 17 \text { (stat. }) \pm 2\left(\text { bin-by-bin }{ }^{2}\right) \pm 1(\text { syst. }) \pm 1(\text { theory })\right)^{\circ}
$$

where the uncertainty is split into its various contributions. As shown in the right part of Fig. 3 a pure CP-odd hypothesis $\left(\kappa_{\tau}=0, \tilde{\kappa}_{\tau}=1\right)$ is excluded at $99.7 \%$ confidence level.
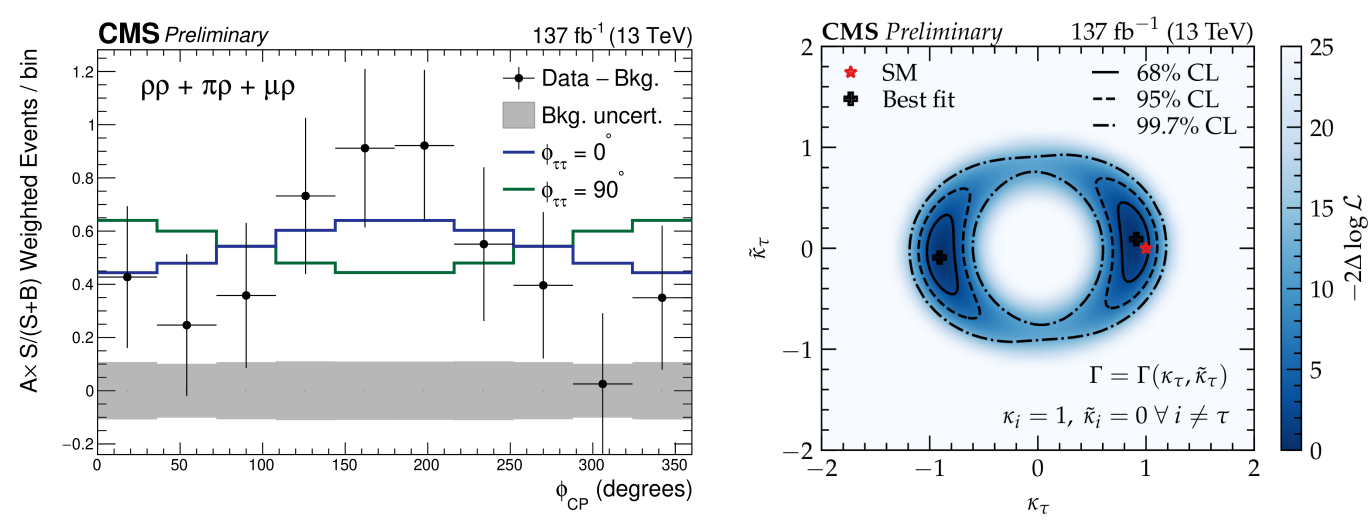

Figure 3: Left: weighted acoplanarity angle distribution in recorded data after subtracting the expected background. Right: negative log-likelihood scan with respect to the pure CP-even and CP-odd Yukawa couplings [4].

No deviation from the SM expectation was observed. This limits the allowed amount of CP violation in the Higgs sector, and can be used to constrain the allowed phase space for theories beyond the SM $[7,8]$.

\footnotetext{
${ }^{2}$ Bin-by-bin uncertainty of signal and background models, representing the effect of the limited statistics for MC simulation and data-driven background estimation.
} 


\section{References}

[1] ATLAS Collaboration, Observation of a new particle in the search for the Standard Model Higgs boson with the ATLAS detector at the LHC, Physics Letters B 716 (2012), no. 1, 1 - 29, doi:10.1016/j.physletb.2012.08.020.

[2] CMS Collaboration, Observation of a new boson at a mass of $125 \mathrm{GeV}$ with the CMS experiment at the LHC, Physics Letters B 716 (2012), no. 1, 30 - 61, doi:10.1016/j.physletb.2012.08.020.

[3] A. Cardini, Measurement of the CP properties of the Higgs boson in its decays to $\tau$ leptons with the CMS experiment, PUBDB-2021-03550, DESY-THESIS-2021-015, 10.3204/PUBDB2021-03550.

[4] CMS Collaboration, Analysis of the CP structure of the Yukawa coupling between the Higgs boson and $\tau$ leptons in proton-proton collisions at $\sqrt{s}=13 \mathrm{TeV}$, CMS-PAS-HIG-20-006, cds.cern.ch/record/2725571.

[5] CMS Collaboration, Performance of the DeepTau algorithm for the discrimination of taus against jets, electron, and muons, CMS-DP-2019-033, cds.cern.ch/record/2694158.

[6] CMS Collaboration, Identification of hadronic tau decay channels using multivariate analysis (MVA decay mode), CMS-DP-2020-041, cds.cern.ch/record/2727092.

[7] S. F. King, M. Muhlleitner, R. Nevzorov, and K. Walz, Exploring the CP-violating NMSSM: EDM Constraints and Phenomenology, Nucl. Phys. B 901 (2015) 526-555, doi:10.1016/j.nuclphysb.2015.11.003.

[8] S.-F. Ge, G. Li, P. Pasquini, and M. J. Ramsey-Musolf, CP-violating Higgs Di-tau Decays: Baryogenesis and Higgs Factories, doi:10.1103/PhysRevD.103.095027. 